\title{
Factores de riesgo obstétrico en pacientes recibidas para atención del parto en dos instituciones de primer nivel en Santafé de Bogotá, Colombia, 1993
}

\author{
María Inés Sarmiento Medina*
}

RESUMEN: Se revisaron 249 casos que solicitaron atención del parto en dos instituciones de primer nivel en Bogotá (Colombia). Se evaluó su clasificación segun factores de riesgo y su evolución inmediata.

Contrariamente a lo que se esperaba, solamente el $34 \%$ de los casos atendidos fueron clasificados como de bajo riesgo. El principal problema detectado fue la falta de información para lograr una valoración adecuada del riesgo. En consecuencia, los factores de riesgo no se tuvieron en cuenta para definir el nivel de atención de los casos segun su complejidad. Hubo $44 \%$ de omisión en la clasificación.

Aunque se atendieron partos de riesgo superior al primer nivel, las complicaciones no requirieron intervención de niveles más especializados y no se registraron muertes maternas ni fetales.

Se cuestiona la efectividad de la estrategia de atención obstétrica por niveles de complejidad mientras no haya rigurosidad en los mecanismos de clasificación y una definición precisa de la capacidad de resolución de cada nivel.

PAlabras ClaveS: Factores de Riesgo Obstétrico, Primer Nivel de Atención, Calidad de la Atención en Salud.

SUMMARY: 249 Clinic Files of deliveries in two Primary Health Care Institutions in Bogotá (Colombia) were reviewed. Their classification according to risk factors and their inmediate evolution were evaluated.

Only $34 \%$ of the cases has been classified as low risk. The main problem detected was the insufficient information in order to obtain an adequate level of risk. Consequently, the risk factors were not utilized in order to refer patients according to their risk level. As much as $44 \%$ of the cases were nor classified.

Althoug some deliveries wich risk was higher than assistance in Primary Health Care, the complications did not require any reference to higher levels. No maternal or fetal deads were registered.

The effectiveness of the obstetrical assistance strategy according to the complexity level is questioned until the risk classification factors and the resolution capacity definition of each level have been not rigourosly established.

KEY WORDS: Obstetrical Risk Factors, Primary Health Care Quallity of health assistance.

La mortalidad materna en Latinoamérica y el Caribe asciende a cerca de 28.000 personas por año como consecuencia de abortos, complicaciones del embarazo, parto y postparto (17). Determinantes económicos, sociales y demográficos tienen gran importancia en la magnitud de las muertes de madres y niños menores de 1 año, y son reflejo de la calidad de vida en los paises (8). La inequidad y la desigualdad en los servicios de salud ahondan la brecha y marcan grandes diferencias a nivel de clases sociales y regiones así como entre unos paises y otros $(3,13)$.

La atención por niveles según el riesgo obstétrico se ha propuesto desde hace varios años como una alternativa tendiente a racionalizar el uso de los recursos y ofrecer

MD. Especialista en Epidemiología. Profesora Asistente. Departamento de Medicina Preventiva, Universidad Nacional de Colombia. mayor cobertura de asistencia institucional del parto, con el fin de disminuir dicha morbimortalidad (11).

Estudios epidemiológicos han demostrado asociaciones de la morbilidad y mortalidad materna con factores relacionados con antecedentes de la madre. Algunos de ellos como por ejemplo la procedencia rural, la edad extrema de la madre, el número de partos anteriores, el espaciamiento codo entre embarazos, el parto prematuro previo, el hábito de fumar, la ingesta de drogas o alcohol durante el embarazo, la ausencia de atención prenatal o el bajo nivel educativo de la madre han mostrado asociaciones estadísticamente significativas con el bajo peso del recién nacido, el bajo puntaje en la valoración neurofisiológica en el momento del nacimiento, la morbilidad perinatal y el aborto espontáneo $(3,7,9-10,17)$.

El concepto epidemiológico de riesgo conlleva la detección de los factores asociados de manera temprana para intervenir oportunamente con el fin de evitar com- 
plicaciones o predecirlas para estar preparado para cuando ellas sucedan $(1,3-4,6)$. Con base en él, se han diseñado estrategias de atención maternoinfantil y se han elaborado instrumentos que permitan cuantificar y evaluar el riesgo prenatal con el fin de atender a las pacientes en el nivel tecnológico que les corresponda de acuerdo a la complejidad de su embarazo $(2-3,9-10,12,14,17)$.

Con esta estrategia se espera que un parto de bajo riesgo pueda ser atendido por médicos generales, enfermeras o parteras capacitadas y uno de alto riesgo se remita a instituciones con alta tecnología y personal especializado (15). Los embarazos de riesgo constituyen el $20 \%$ de todos los embarazos y son responsables del 70 a $80 \%$ de toda la morbilidad perinatal (10).

Si bien la racionalidad de esta estrategia es bastante evidente desde el punto de vista administrativo con el fin de utilizar los recursos de manera óptima, su eficacia se cuestiona si hay fallas en la clasificación del riesgo, si el sistema de remisión y contraremisión entre los diferentes niveles no está funcionando correctamente o si las instituciones no están suficientemente dotadas, capacitado su personal y definido claramente el alcance de su capacidad resolutiva. En tales casos asume el médico un alto riesgo sin la infraestructura necesaria que le permita evitar complicaciones maternas o fetales que pueden llegar incluso hasta la muerte de uno de ellos o de ambos.

En este estudio se hizo una revisión de las características de los partos atendidos en dos instituciones de primer nivel, donde se espera se atiendan partos de baja complejidad. Se exploró la presencia de factores de riesgo, la clasificación del caso al ingreso, el desarrollo del parto y su evolución inmediata, con el fin de hacer una evaluación de la estrategia de atención por niveles mediante el enfoque de riesgo.

\section{Objetivos}

1. Conocer la frecuencia de factores de riesgo presentes en las mujeres que consultaron el primer nivel para atención del parto.

2. Conocer la frecuencia con la cual se asumieron riesgos superiores al nivel en el que se estaba atendiendo el parto.

3. Describir el tipo de complicaciones que se presentaron.

4. Evaluar la dinámica del sistema de remisión y contraremisión entre los diferentes niveles.

\section{Metodología}

Se realizó un estudio prospectivo revisando 249 partos atendidos durante un trimestre en las dos instituciones de primer nivel de uno de los Sistemas Locales de Salud (SILOS ) de Santafé de Bogotá, Distrito Capital. Se tomó la información consignada en la historia clínica y los libros de registro de la sala de partos y se complementó con una entrevista con las pacientes para solicitar información que no se había registrado en la historia clínica.

La institución de primer nivel se definió de acuerdo al Sistema Distrital de Urgencias (5) y denominada Centro de Atención Médica Inmediata de Primer Nivel, CAMI 1 y su infraestructura es bastante homogénea en los 17 centros de este nivel que hay en Bogotá. Cuentan con un profesional médico general, o médico en servicio social obligatorio apoyado por una o dos auxiliares de enfermería, quienes cubren toda la demanda de urgencias por consulta general y por obstetricia durante las 24 horas del día. Posee una sala de partos dotada con instrumental básico (máximo dos o tres equipos completos de instrumental quirúrgico para la atención de partos), succionador, ambú y laringoscopio para recién nacidos y adultos. La ropa quirúrgica es escasa y no se utiliza de rutina en las pacientes. Algunos de estos centros de atención tienen una incubadora. Además hay un radioteléfono que se comunica con el nivel central de la Secretaría de salud del distrito capital, y con las diferentes instituciones de la red de urgencias y tiene también una ambulancia.

El Centro de Atención Médica Inmediata de primer nivel recibe los partos de pacientes remitidas de los centros de salud en los que se ha hecho la atención prenatal, los remitidos por médico particular y además los que no son remitidos y llegan por decisión voluntaria de las pacientes en el momento del trabajo de parto.

Con base en la revisión bibliográfica y especialmente dos estudios anteriores realizados en Bogotá $(2,12)$, se elaboró un patrón que definiera un parto de bajo riesgo ideal, y se comparó con los casos atendidos. Sus características son las siguientes :

1. Edad materna entre 18 y 34 años

2. Paridad entre 1 y 4 hijos

3. Edad gestacional entre 37 y 41 semanas.

4. Control prenatal de inicio en el primero o segundo trimestre con 4 visitas como mínimo en todo el transcurso de la gestación.

5. Con serología negativa para infección sifilítica y hemoclasificación correspondiente a Rh positivo. Glicemia normal, anticuerpos para toxoplasmosis negativos o bajos al menos en dos controles.

6. Con una ecografía obstétrica por lo menos en el último trimestre que permita confirmar edad gestacional, tamaño adecuado del feto, situación longitudinal y presentación cefálica.

7. Antecedentes obstétricos negativos para:

- Muerte perinatal menor de 8 días de nacido o mortinato

- Más de dos abortos espontáneos sucesivos

- Cesárea previa

- Cirugía vaginal o cervical

- Espacio intergenésico menor de dos años

8. Con las siguientes condiciones al examen de ingreso:

- Sin patología general severa detectable

- Temperatura menor de 37.6 grados centígrados

- Tensión arterial diastólica menor de $90 \mathrm{~mm} \mathrm{hg}$

- Altura Uterina entre 30 y $34 \mathrm{~cm}$

- Feto único, vivo con fetocardia entre 120 y 160 latidos por minuto

- Situación longitudinal y presentación cefálica

- Membranas íntegras o ruptura menor de 8 horas con líquido claro.

Teniendo en cuenta que el enfoque de riesgo se basa en la presencia o ausencia de una serie de factores, la falta de información en el momento de la anamnesis se consideró como sinónimo de riesgo. Es decir, mientras no se 
confirme la ausencia de uno de ellos, se está asumiendo un riesgo potencial por la probabilidad de su presencia, la cual implica dejar de tomar una serie de precauciones que podrían evitar una eventual complicación.

Se evaluó cada período del parto. Se analizaron todas las complicaciones de diferente gravedad y también se exploró el uso de medicamentos en la madre o en el niño con el fin de tener un parámetro indirecto de evaluación del desarrollo del parto.

\section{Resultados}

Se atendieron 249 consultas por trabajo de parto, de las cuales fueron remitidas $44(17.4 \%)$ porque según el criterio del médico debían ser atendidas en instituciones de niveles superiores. Tres de ellas no pudieron ubicarse por falta de camas, por lo cual tuvieron que ser atendidas en el primer nivel.

Los diagnósticos de remisión se pueden observar en la tabla 1:

Tabla 1

DIAGNOSTICOS DE REMISION DE LAS PACIENTES

QUE CONSULTARON AL CENTRO DE PRIMER NIVEL, SANTAFE DE BOGOTA 1994

\begin{tabular}{|lcc|}
\hline Diagnóstico & Número & $\%$ \\
\hline Parto pre-término & 9 & 20.5 \\
Ruptura prolongada de membranas & 5 & 11.4 \\
Pre-eclampsia & 3 & 6.8 \\
Presentación anómala & 2 & 4.5 \\
Sufrimiento fetal agudo & 2 & 4.5 \\
Otras & 23 & 52.3 \\
Total & 44 & 100 \\
\hline
\end{tabular}

El número total de pacientes cuyo parto fue atendido en el primer nivel durante el trimestre del estudio fue de 205 , correspondiente al $82.6 \%$ de los casos que consultaron por este motivo.

La tabla 2 muestra la frecuencia de factores de riesgo en el total de pacientes atendidas en la institución de primer nivel. Si se suman las columnas 3 y 4 se puede ver la cantidad de pacientes que fueron atendidas con riesgo ya sea explícito o por desconocimiento, el cual viene a ser muy alto en especial en algunos grupos de factores como los sociodemográficos y la atención prenatal. Otros factores llaman la atención no tanto por su alta frecuencia como por su importancia clínica, como la tensión arterial, la altura uterina y la edad gestacional.

En el gráfico 1 se puede observar la frecuencia con la cual se presentaron los factores evaluados como causantes de riesgo en las pacientes atendidas en el primer nivel. Las frecuencias más altas se encuentran en los relacionados con la atención prenatal, y los antecedentes ginecoobstétricos.

El gráfico 2 muestra la frecuencia de omisión de los diferentes factores de riesgo en el momento de la valoración de la paciente en la institución de primer nivel.

En relación con las características sociodemográficas como procedencia, estado civil o escolaridad no fueron considerados en la anamnesis en 144 de los casos (70\%). El $17 \%$ de las pacientes presentó riesgo debido al factor edad.

Aspectos relacionados con la ausencia de control prenatal adecuado y oportuno fueron causa de riesgo en más del $52 \%$ de los casos. Si se tiene en cuenta la ausencia de información, se alcanza al $88 \%$ como en el caso del número de controles prenatales.

Tabla 2

RIESGO EN 205 PACIENTES OBSTETRICAS ATENDIDAS EN INSTITUCIONES DE PRIMER NIVEL SEGUN CUATRO GRUPOS DE FACTORES ESTUDIADOS. *

\begin{tabular}{|l|cc|cc|cc|c|}
\hline \multicolumn{1}{|c|}{ Factor } & \multicolumn{2}{|c|}{$\begin{array}{c}\text { Fuera de } \\
\text { Riesgo }\end{array}$} & \multicolumn{2}{c|}{$\begin{array}{c}\text { Sin } \\
\text { Información }\end{array}$} & \multicolumn{2}{c|}{ En riesgo } & Total \\
\hline & No. & \% & No. & $\%$ & No. & \% & Total No. \\
Procedencia & 58 & 28.3 & 144 & 70.3 & 3 & 1.4 & 205 \\
Estado Civil & 40 & 19.5 & 140 & 68.3 & 25 & 12.2 & 205 \\
Escolaridad & 4 & 1.9 & 195 & 95.2 & 6 & 3.1 & 205 \\
Edad & 162 & 79.4 & 8 & 3.9 & 35 & 17.2 & 205 \\
Atención Prenatal & 92 & 45.1 & 50 & 24.5 & 57 & 27.93 & 205 \\
Remisión por personal de salud & 67 & 32.8 & 13 & 6.4 & 111 & 54.4 & 205 \\
Inicio del control prenatal & 33 & 16.8 & 168 & 82.3 & 4 & 2 & 205 \\
Número de controles prenatales & 29 & 14.2 & 168 & 82.3 & 8 & 4 & 205 \\
Número de embarazos123 & 60 & 0 & 0 & 82 & 40 & 205 & \\
Número de abortos & 172 & 84.3 & 0 & 0 & 33 & 16.2 & 205 \\
Espacio intergenésico & 20 & 9.8 & 148 & 72.5 & 37 & 18.1 & 205 \\
Patologías durante el embarazo & 143 & 70.7 & 45 & 22.1 & 0 & 0 & 205 \\
Exámenes de laboratorio & 144 & 70.1 & 0 & 0 & 61 & 29.9 & 205 \\
Ecografías & 100 & 49 & 23 & 11.3 & 82 & 40.2 & 205 \\
Tensión arterial & 188 & 92.1 & 12 & 5.4 & 5 & 2.5 & 205 \\
Edad gestacional & 171 & 83.7 & 18 & 8.7 & 16 & 7.6 & 205 \\
Altura uterina & 142 & 69.4 & 18 & 8.7 & 45 & 22 & 205 \\
\hline
\end{tabular}


Gráfico 1

\section{FACTORES DE RIESGO OBSTETRICO EN 205 PACIENTES ATENDIDAS *}

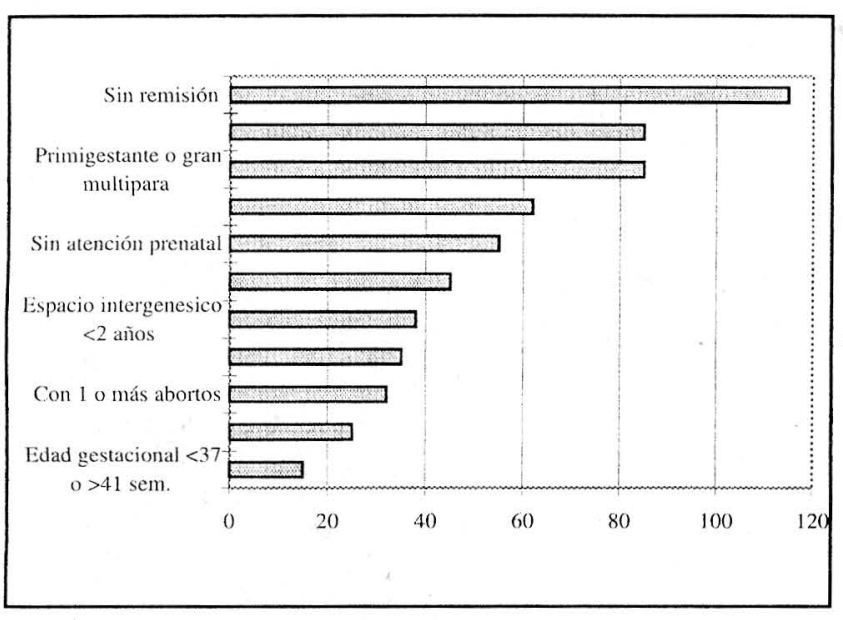

Gráfico 2

FRECUENCIA DE FACTORES OMITIDOS EN LA VALORACION DE, 205 PACIENTES OBSTETRICAS ATENDIDAS *

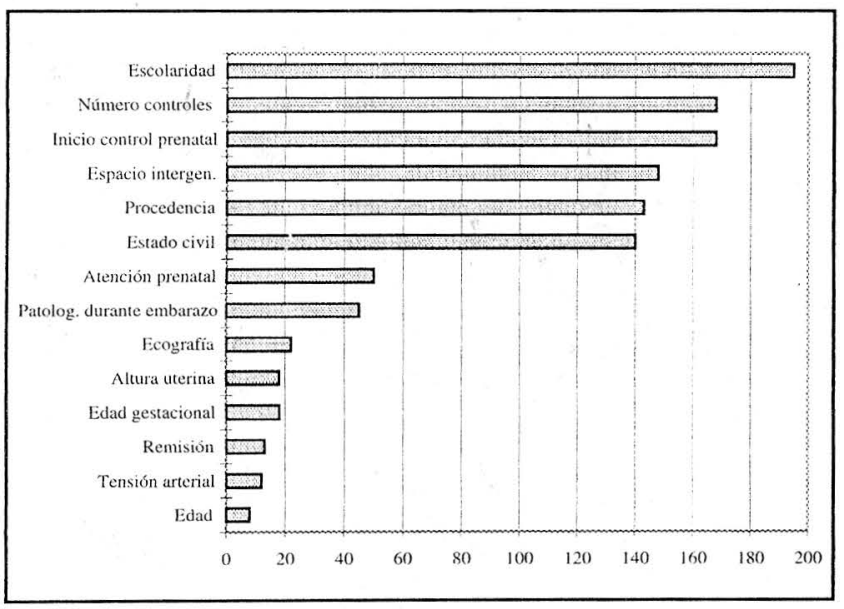

Con espacio intergenésico menor de 2 años se presentaron 37 pacientes (18\%). Si a ello se suma la ausencia de información, se llega a 185 pacientes $(91 \%)$ en las que se asumió riesgo por esta causa.

Respecto a los antecedentes gineco-obstétricos, se encontraron en riesgo por ser primigestantes o grandes multíparas $40 \%$ de las pacientes.

Con edad gestacional en riesgo por pre-término o post-término se encontraron 16 pacientes correspondientes al $7.6 \%$ y con altura uterina anormal para la edad gestacional por exceso o defecto estuvieron en riesgo 45 pacientes $(22 \%)$.
Las patologías leves concomitantes con el embarazo fueron várices, hemorroides, infección vaginal no venérea, infección urinaria y desnutrición. Fueron registradas por el médico en un $70 \%$ de los casos que las padecían. No se encontraron patologías severas.

Con tensión arterial diastólica mayor de $90 \mathrm{~mm} \mathrm{Hg} \mathrm{o}$ ausencia de información al respecto se encontraron en riesgo 17 pacientes atendidas $(8.5 \%)$.

En el gráfico 3 se puede observar la clasificación del riesgo que el médico hizo en cada caso con base en la remisión, la anamnesis, el examen físico y la revisión de exámenes paraclínicos. Se observa como solamente 70 pacientes $(34 \%)$ fueron clasificadas explícitamente como de bajo riesgo obstétrico, lo cual significa que el $66 \%$ de los casos fueron atendidos en el primer nivel asumiendo un riesgo potencial de complicaciones.

En la tabla 3 se observan las causas por las cuales los médicos clasificaron el parto como alto o mediano riesgo. Llaman la atención las causas de riesgo como pre-eclampsia, embarazo múltiple, edad gestacional menor de 37 semanas y sufrimiento fetal agudo, porque requieren de manejo especializado en cuanto a recurso humano $y$ tecnológico, de los cuales se carece en los centros de primer nivel.

Tabla 3

CAUSAS DE RIESGO REGISTRADAS EN LA HISTORIA CLINICA DE PACIENTES ATENDIDAS *

\begin{tabular}{|c|c|c|}
\hline Causa & Número & $\begin{array}{c}\text { Porcentaje sobre } \\
205 \text { partos } \\
\text { atendidos }\end{array}$ \\
\hline Primigestante & 18 & 8.8 \\
\hline $\begin{array}{l}\text { Ruptura Prolongada } \\
\text { de membranas }\end{array}$ & 7 & 3.4 \\
\hline Sin Control Prenatal & 6 & 2.9 \\
\hline $\begin{array}{l}\text { Edad gestacional mayor } \\
\text { de } 37 \text { o menor de } 42 \\
\text { semanas }\end{array}$ & 2 & 1 \\
\hline Preeclampsia & 2 & 1 \\
\hline Madre Rh negativo & 2 & 1 \\
\hline $\begin{array}{l}\text { Altura uterina mayor } \\
\text { de } 33 \text { o menor de } 30 \mathrm{~cm}\end{array}$ & 1 & 0.5 \\
\hline $\begin{array}{l}\text { Edad materna mayor de } \\
18 \text { o mayor de } 36 \text { años }\end{array}$ & 1 & 0.5 \\
\hline Gran multípara & 1 & 0.5 \\
\hline Embarazo múltiple & 1 & 0.5 \\
\hline Pelvis Estrecha & 1 & 0.5 \\
\hline Sufrimiento fetal Agudo & 1 & 0.5 \\
\hline Total & 37 & 18.04 \\
\hline
\end{tabular}


Gráfico 3

VALORACION DEL RIESGO EN PACIENTES OBSTETRICAS ATENDIDAS*

\section{Alto o medio}

$22 \%$

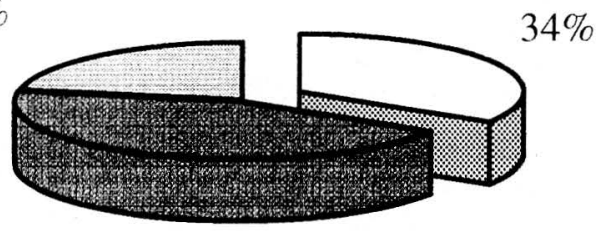

$\operatorname{Sin}$

información

$44 \%$
Las complicaciones que se encontraron con mayor frecuencia fueron sufrimiento fetal agudo en $15 \%$ de los casos, (31 pacientes), desgarro perineal en 13.7\% (28 casos), hemorragia en $5.4 \%$ ( 11 casos), irregularidad en el trabajo de parto en $3.4 \%$ ( 7 casos).

Su manejo fue en su mayoría con medicamentos. Se utilizaron oxcitócicos en el $20 \%$ de los partos atendidos y antibióticos en un 14\%. Los desgarros perineales fueron suturados. Se realizaron 13 revisiones manuales de cavidad uterina, lo cual corresponde al $6.3 \%$ de los partos atendidos. Solamente 3 de ellas se efectuaron bajo el efecto de anestesia disociativa. El 1.5\% de los partos atendidos fueron remitidos a niveles superiores para el manejo de las complicaciones, 2 de ellos por desgarro de cuello uterino y 1 por dificultad respiratoria del recién nacido.

No se registró ninguna muerte perinatal ni materna durante la atención en el primer nivel. El peso al nacer del producto de los partos atendidos estuvo entre 2700 y 3500 gramos en 170 casos (83\%). Por debajo de $2700 \mathrm{gm}$ estuvieron 17 casos, y un número igual por encima de $3500 \mathrm{gm}$.

\section{Discusión}

La atención del parto por niveles de complejidad exige gran precisión y confiabilidad en la clasificación de su riesgo, más aún, se fundamenta en dicha clasificación, la cual define el tipo de institución y de personal que atenderá el parto para garantizar las condiciones óptimas en cada caso.

No obstante ser éste un estudio de tipo exploratorio y con inferencia limitada, se puede concluir que la estrate- gia de atención por niveles de complejidad no está siendo utilizada de manera adecuada. Se evaluó la cuarta parte de los partos atendidos en un año en dos instituciones de primer nivel y se encontró que no se está cuantificando el riesgo de manera sistemática y se está subestimando en gran medida la presencia de factores que han dado evidencia clínica y epidemiológica de asociación con complicaciones de la madre y el niño. Esto se refleja en el alto porcentaje de pacientes en las que se omitió la clasificación de riesgo en el momento de ingresar al centro de primer nivel.

Teniendo en cuenta la rápida evolución del caso obstétrico, es necesario contar con suficientes elementos de la anamnesis y del examen físico para la toma de decisiones oportunas. Sinembargo, la ausencia de información en la historia clínica fue el problema más frecuentemente encontrado en este estudio.

Se podría pensar que una cantidad de trabajo excesivo sea una de las causas por las que no se esté haciendo una evaluación detallada de cada paciente, no obstante no parece ser éste el caso, puesto que en las dos instituciones se atendieron únicamente 3 pacientes al día en promedio durante el trimestre evaluado. También una falla frecuente del sistema de recepción en el tercer nivel podría provocar que el médico del primer nivel considere que la clasificación del riesgo es una tarea sin sentido, pues debe atender a todas las pacientes en el primer nivel, independientemente de su complejidad. No obstante, en este estudio se encontró que la demanda insatisfecha en el tercer nivel fue solamente del $7 \%$ (correspondiente a 3 de 44 casos remitidos).

Se hace necesario insistir en la elaboración de una historia clínica completa y diseñar instrumentos ágiles que orienten el interrogatorio y el examen físico para evitar omisiones y cuantificar el riesgo de cada paciente. Se recomienda el uso de instrumentos para su clasificación en tres momentos en los que se toman decisiones que trascienden a períodos posteriores: durante el control prenatal, en el momento de la remisión para la atención del parto y durante la admisión de la paciente en trabajo de parto.

Involucrar a médicos y enfermeras que están directamente relacionados con la atención de partos en los diferentes niveles, en el diseño de los instrumentos y la evaluación de la calidad de la atención obstétrica puede ser una estrategia que genere compromiso y probablemente provoque en ellos cambios de actitudes y conductas de manera más duradera.

El sistema de atención por niveles de complejidad debe operar con precisión en lo que se refiere a la capacidad resolutiva de cada nivel. Como este trabajo lo demuestra, una importante proporción de los partos atendidos en el primer nivel no son de bajo riesgo. Por esta razón se hace necesario aplicar una mayor rigurosidad en la selección de las pacientes o una dotación con recursos humanos y técnicos adecuados para satisfacer, en el primer nivel, demandas similares a las encontradas en las instituciones estudio. 


\section{BIBLIOGRAFIA}

1. Aubry RH., Pennington JC. ldentification and evaluation of high-risk pregnancy: The perinatal concept. Clin Obstet Gyn 1973; 16: 3-27.

2. Castrillón María Consuelo Escobar I., Pulido S. Calidad de la atención en salas de trabajo de parto. Instituciones oficiales. Antioquia, Colombia 1990. Rev Col de Obst y Gin 1992; 43 (1).

3. Cataño O. Mortalidad Materna y Factores de Riesgo Asociados. Estudio de casos y controles. Rev Col de Obst y Gin 1992; 43 (2).

4. Cifuentes R. Obstetricia de Alto Riesgo. Ediciones Aspromédica. Cali, Colombia. Cuarta Edición 1994.

5. Concejo de Santafé de Bogotá D.C., Acuerdo No. 16 de 1991. Sistema Distrital de Atención de Urgencias.

6. Coopland AT., Peddle LJ., Baskett TF et al. A simplified antepartum high-risk pregnancy screening form: Statistical analysis of 5459 cases. Can Med Assoc J 1977; 116: 999 - 1001.

7. De la Cruz Jairo. Modalidad Perinatal en el Quinquenio 1985 a 1989 en la Sección de Ginecología y Obstetricia del Hospital Militar Central. Rev Col Obst y Gin 42 (1).

8. Dott AB., Fort AT. The effect of maternal demographic factors on infant mortality rates: summary of the findings of the Louisiana infant mortality study, Part 1 . Am J Obst Gyn 1975; 123: 847.

9. Edwards LE. A simplified antepartum risk score system. Obst and Gyn 1979; 54 (2).
10. Hobel CJ., Hyvarinen MA., Okadda DM et al. Prenatal and intrapartum high-risk screening. Am J Obst Gyn 1973; 117: 1.

11. Organización Panamericana de la Salud. Manual sobre el enfoque de riesgo en la atención materno-infantil. Serie Paltex para ejecutores de programas de salud No 71985.

12. Pazmiño de Osorio Sonia. Evaluación tecnológica del parto de bajo riesgo en diferentes modelos de atención. Resultados del niño y costos. Rev Col de Obst Gin. 51(3).

13. Programa de Naciones Unidas Para el Desarrollo PNUD. Informe sobre Desarrollo Humano, 1994.

14. Quintero C., Rodríguez J., Muñoz R. Clínica del Riesgo Obstétrico. Rev Col de Obst Gin 1975; 36 (1).

15. Schwarcz R. y col. Historia clínica perinatal simplificada. Propuesta de un modelo para la atención primaria de baja complejidad. OPS OMS CLAP. Publicación Científica No. 973, 1983.

16. Sokol R., Mortimer G., Stojkov J. Clinical applications of high risk scoring on an obstetric service. Am J Obstet and Gynecol 1977; 128 (6).

17. Sotelo F. Juan. El enfoque de riesgo y la mortalidad materna: una perspectiva latinoamericana. Boletín de la Oficina Sanitaria Panamericana $1993 ; 114(4)$. 